\title{
On the approximation of electronic wavefunctions by anisotropic Gauss and Gauss-Hermite functions
}

\author{
Stephan Scholz · Harry Yserentant
}

November 27, 2016

\begin{abstract}
The electronic Schrödinger equation describes the motion of $N$ electrons under Coulomb interaction forces in a field of clamped nuclei. The solutions of this equation, the electronic wavefunctions, depend on $3 N$ variables, three spatial dimensions for each electron. We study the approximability of these wavefunctions by linear combinations of anisotropic Gauss functions, or more precisely Gauss-Hermite functions, products of polynomials and anisotropic Gauss functions in the narrow sense. We show that the original, singular wavefunctions can up to given accuracy and a negligibly small residual error be approximated with only insignificantly more such terms than their convolution with a Gaussian kernel of sufficiently small width and that basically arbitrary orders of convergence can be reached.
\end{abstract}

Mathematics Subject Classification (2000) 35J10 · 41A25 · 41A63

\section{Introduction}

The approximation of high-dimensional functions, whether they be given explicitly or implicitly as solutions of differential equations, represents one of the grand challenges of applied mathematics. This field made great progress during the past years, above all by the emergence of modern tensor product methods [10]. The astonishing efficiency of such methods for the numerical solution of certain partial differential equations and their obviously often rapid convergence can meanwhile be explained theoretically [8]. Adaptive techniques [2] have been developed that enable to exploit this convergence behavior in practical computations. One of the most notorious and complicated problems of this type, however, the electronic Schrödinger equation, largely resists such approaches. The Schrödinger equation forms the basis of quantum mechanics and is of fundamental importance for our understanding of atoms and molecules. It links chemistry to physics and describes a system of electrons and nuclei that interact by Coulomb attraction and repulsion forces. As proposed by Born

Institut für Mathematik, Technische Universität Berlin, 10623 Berlin, Germany

E-mail: stscholz@math.tu-berlin.de,yserentant@math.tu-berlin.de 
and Oppenheimer in the nascency of quantum mechanics, the slower motion of the nuclei is mostly separated from that of the electrons. This results in the electronic Schrödinger equation, the problem to find the eigenvalues and eigenfunctions of the electronic Hamilton operator 1

$$
H=-\sum_{i=1}^{N} \Delta_{i}-\sum_{i=1}^{N} \sum_{v=1}^{K} \frac{Z_{v}}{\left|x_{i}-a_{v}\right|}+\frac{1}{2} \sum_{\substack{i, j=1 \\ i \neq j}}^{N} \frac{1}{\left|x_{i}-x_{j}\right|} .
$$

It acts on functions with arguments $x_{1}, \ldots, x_{N}$ in $\mathbb{R}^{3}$, which are associated with the positions of the considered electrons. The $a_{1}, \ldots, a_{K}$ in $\mathbb{R}^{3}$ are the fixed positions of the nuclei and the values $Z_{v}>0$ the charges of the nuclei in multiples of the electron charge. The reason for the comparatively low performance of tensor product methods when applied to the electronic Schrödinger equation is that such methods fix a set of directions. It is not possible with tensor product methods to capture simultaneously and equally well the singularities arising from the interaction of the electrons and the nuclei aligned with the coordinate directions and from the electron-electron singularities aligned with the diagonals.

Therefore we come back to an old idea, the approximation of the eigenfunctions of the Schrödinger operator (1.1), the electronic wavefunctions, by Gauss functions. The almost exclusive use of Gauss functions in quantum chemistry [11] is partly motivated by the fact that the arising integrals can be easily evaluated but has also to do with their good approximation properties. We study the approximability of electronic wavefunctions by linear combinations of Gauss-Hermite functions

$$
P(x) \exp \left(-\frac{1}{2}(x-a) \cdot Q(x-a)\right)
$$

composed of a polynomial part $P(x)$ and an anisotropic Gauss function. We will denote such functions in what follows shortly as Gauss functions. The symmetric positive definite matrices $Q$ are arbitrary and not fixed in advance. The same holds for the points $a \in \mathbb{R}^{3 N}$ around which the Gauss functions are centered and which are only indirectly determined by the positions of the nuclei. The ansatz in particular covers products of Gaussian orbitals and Gaussian geminals. The key point is that the set of these functions is invariant to linear transformations and shifts of the coordinate system. There is therefore no need to distinguish between the singularities arising from the interaction of the electrons and the nuclei and among the electrons themselves. Basically we show that electronic wavefunctions can be approximated with arbitrary order in the number of the involved terms by linear combinations of such Gauss functions. This behavior is in contrast to approximation results that are based on the mixed regularity of the wavefunctions; see [19,20,21] and [13]. These regularity properties together with the antisymmetry of the wavefunctions enforced by the Pauli principle suffice to construct approximations by linear combinations of Slater determinants composed of a fixed set of basis functions that converge with an order in the number of the involved terms that does not deteriorate with the number of

\footnotetext{
1 To simplify the presentation, we omit the usual factor $1 / 2$ in front of the kinetic energy part, which corresponds only to a minor change of the lengthscale and does not affect the mathematics.
} 
electrons. The attainable convergence order is, however, fundamentally limited by the limited regularity of the wavefunctions. In the present work, we do not make direct use of regularity properties of the wavefunctions. The approximations are constructed directly by a kind of iterative procedure.

The key to our approximation of the wavefunctions is the extremely accurate approximation of the functions $1 / \sqrt{r}$ and $1 / r$ by exponential functions and with that indirectly also that of $1 / r$ by Gauss functions. Approximations of this kind form a rather universal tool that received much attention during the past years. Braess [5] and Kutzelnigg [14] studied such approximations in view of applications in quantum mechanics, particularly regarding the hydrogen ground state. Bachmayr, Chen, and Schneider [1] extended this work to excited states. Braess and Hackbusch [6] have shown that the best uniform approximation of $1 / r$ on intervals $[R, \infty), R>0$, by finite linear combinations of exponential functions converges almost exponentially in the number of the involved terms. A detailed exposition of such approximations and results and a survey on some of their applications can be found in [7].

The central idea is to approximate the Coulomb potentials in the operator 1.1) and the inverse of the correspondingly shifted Laplace operator, expressed in terms of the Fourier transform, with very high relative accuracy by series of Gauss functions. The original eigenvalue problem is at first rewritten as a linear equation with the convolution $f=K * u$ of the eigenfunction $u$ under consideration with a Gaussian kernel $K$ of sufficiently small width as right hand side. The solution of the corresponding approximate equation with same right hand side is then orders of magnitude closer to the solution $u$ of the original equation than $u$ to the true physical wavefunction influenced, among other things, by relativistic effects and spin-orbit coupling. The approximate equation is solved via a Neumann series. This series is, after expansion of the right hand side into a series of Gauss functions, itself a series of Gauss functions that is truncated in an appropriate manner. The main hurdle, to which most of this paper is devoted, is the control of this truncation process.

The main result can be roughly sketched as follows. Assume that there exists an infinite sequence $g_{1}, g_{2}, \ldots$ of Gauss functions such that for every $\varepsilon>0$

$$
\left\|K * u-\sum_{j=1}^{n} g_{j}\right\|_{1} \leq \varepsilon, \quad n \leq\left(\frac{\kappa}{\varepsilon}\right)^{1 / r},
$$

where $r$ is a given approximation order and the norm is the $H^{1}$-norm, the norm associated with the Schrödinger equation. Such expansions can be constructed using the exponential decay of the wavefunctions, and when indicated also of their integer and fractional order mixed derivatives [13, 20,21]. The solution of the approximate equation, which serves as a quasi-exact substitute of the original wavefunction $u$, can then, for arbitrarily small $\varepsilon>0$, be approximated by a linear combination of

$$
n \leq 2\left(\frac{2 \kappa}{\varepsilon}\right)^{1 / r}
$$

Gauss functions of the same polynomial degree up to an $H^{1}$-error $\varepsilon$, provided the width of the smoothing kernel $K$ is sufficiently small in dependence of the approximation order $r$. The approximation of the original, singular wavefunction $u$ up to a very 
small, negligible residual error determined by the approximations of the Coulomb potentials and the inverse of the shifted Laplace operator thus does not require substantially more terms than that of its smoothed variant $K * u$.

The rest of this paper is organized as follows. In Sect.2, the Schrödinger equation is precisely stated, as an eigenvalue problem in weak form on the Sobolev space $H^{1}$. Some properties of its solutions are shortly discussed. More information on the mathematics behind the electronic Schrödinger equation can be found in [20]. In Sect. 3 . the equation is brought into the form from which our approximation result is derived. Sect. 4 discusses the sensitivity of the transformed equation from Sect. 3 to perturbations of the interaction potential and the inverse of the shifted Laplace operator. In Sect. 5] we analyze approximations due to Beylkin and Monzón [4] of the functions $1 / r^{\beta}$ by series of exponential functions. The essential point here is that we have explicit access to the expansion coefficients and that these are intimately connected with a scale of Sobolev norms. These approximations are used in Sect.6 to set up the mentioned approximations of the Coulomb potentials and the inverse of the shifted Laplace operator. Sect. 7 is of rather technical nature and devoted to estimates of the norms of the parts into which the approximations of the single operators split. The key point is that these norms decay exponentially, a property that forms the basis of our final approximation result in Sect. 8

\section{The weak form of the equation}

The solution space of the electronic Schrödinger equation is the Hilbert space $H^{1}$ that consists of the one times weakly differentiable, square integrable functions

$$
u:\left(\mathbb{R}^{3}\right)^{N} \rightarrow \mathbb{R}:\left(x_{1}, \ldots, x_{N}\right) \rightarrow u\left(x_{1}, \ldots, x_{N}\right)
$$

with square integrable first-order weak derivatives. The norm $\|\cdot\|_{1}$ on $H^{1}$ is composed of the $L_{2}$-norm $\|\cdot\|_{0}$ and the $H^{1}$-seminorm $|\cdot|_{1}$, the $L_{2}$-norm of the gradient. The space $H^{1}$ is the space of the wavefunctions for which the total position probability remains finite and the expectation value of the kinetic energy can be given a meaning. By $\mathscr{D}$ we denote the space of all infinitely differentiable functions 2.1 with bounded support. The functions in $\mathscr{D}$ form a dense subset of $L_{2}$ and of $H^{1}$ as well. Before we can state the equation, we have to study the potential

$$
V(x)=-\sum_{i=1}^{N} \sum_{v=1}^{K} \frac{Z_{v}}{\left|x_{i}-a_{v}\right|}+\frac{1}{2} \sum_{\substack{i, j=1 \\ i \neq j}}^{N} \frac{1}{\left|x_{i}-x_{j}\right|}
$$

in the Schrödinger operator (1.1) that is composed of the nucleus-electron interaction potential, the first term in (2.2), and the electron-electron interaction potential.

Lemma 2.1 For arbitrary functions (2.1) in $\mathscr{D}$ and with that also $H^{1}$,

$$
\|V u\|_{0} \leq(2 Z+N-1) N^{1 / 2}|u|_{1},
$$

where $Z=\sum_{v} Z_{v}$ is the total charge of the nuclei. 
The proof of Lemma 2.1 is based on the three-dimensional Hardy inequality

$$
\int \frac{1}{|x|^{2}} v^{2} \mathrm{~d} x \leq 4 \int|\nabla v|^{2} \mathrm{~d} x
$$

for infinitely differentiable functions $v: \mathbb{R}^{3} \rightarrow \mathbb{R}$ with compact support. By 2.3 ,

$$
a(u, v)=\int\{\nabla u \cdot \nabla v+V u v\} \mathrm{d} x=(H u, v)
$$

is a $H^{1}$-bounded bilinear form on $\mathscr{D}$, where $(\cdot, \cdot)$ is the $L_{2}$-inner product. It can be uniquely extended to a bounded bilinear form on $H^{1}$. In this setting, a function $u \neq 0$ in $H^{1}$ is an eigenfunction of the Schrödinger operator (1.1) for the eigenvalue $\lambda$ if

$$
a(u, v)=\lambda(u, v), \quad v \in H^{1} .
$$

The weak form (2.6) of the eigenvalue equation $H u=\lambda u$ in particular fixes the behavior of the eigenfunctions at the singularities of the interaction potential and at infinity. For normed $u, a(u, u)$ is the expectation value of the total energy.

It should be noted that only those eigenfunctions are physically admissible that are antisymmetric with respect to the permutation of the positions $x_{i}$ of electrons of the same spin. This is a consequence of the Pauli principle. We will not utilize this property. We will, however, restrict ourselves to bound states of the system under consideration, eigenfunctions $u$ for eigenvalues $\lambda$ below the ionization threshold, a quantity that is bounded from above by the value zero. Such eigenfunctions and their first order weak derivatives decay exponentially in the $L_{2}$-sense. There is a constant $\mu>0$, depending on the distance of the given eigenvalue $\lambda$ to the ionization threshold, such that the function

$$
x \rightarrow \mathrm{e}^{\mu|x|} u(x)
$$

is square integrable and even possesses square integrable first and certain higher integer and fractional order weak derivatives. This means among other things that the Fourier transforms of these eigenfunctions are real-analytic and that their partial derivatives of arbitrary order are bounded. Such properties and the fact that the eigenvalues under consideration are less than zero will play an essential role in our reasoning. More details, about this and on the electronic Schrödinger equation in general, can be found in [20] and, concerning additional information on the regularity properties of the exponentially weighted wavefunctions, in [13] and [21].

\section{An operator version}

We will fix the eigenvalue $\lambda<0$ under consideration for the rest of this paper and introduce at first the inverse of the correspondingly shifted Laplace operator $-\Delta-\lambda$, a bounded linear operator $G$ from the space $L_{2}$ of the square integrable functions to the Sobolev space $H^{2}$ of the square integrable functions with square integrable first and second order weak derivatives. For rapidly decreasing functions $f$,

$$
(G f)(x)=\left(\frac{1}{\sqrt{2 \pi}}\right)^{3 N} \int \frac{1}{|\omega|^{2}-\lambda} \widehat{f}(\omega) \mathrm{e}^{\mathrm{i} \omega \cdot x} \mathrm{~d} \omega .
$$


For $u \in H^{1}$ and infinitely differentiable functions $v \in \mathscr{D}$, with compact support,

$$
\left(u, G^{-1} v\right)=\int \nabla u \cdot \nabla v \mathrm{~d} x-\lambda(u, v) .
$$

For all square integrable functions $f$ and $g$,

$$
(G f, g)=(f, G g) \text {. }
$$

Lemma 3.1 A function $u \in H^{1}$ solves the eigenvalue equation (2.6) if and only if

$$
u+G V u=0
$$

and is therefore automatically contained in $H^{2}$.

Proof As follows from (3.2) and (3.3), for all $u \in H^{1}$ and all $v \in \mathscr{D}$

$$
\left(u+G V u, G^{-1} v\right)=a(u, v)-\lambda(u, v) .
$$

Since $\mathscr{D}$ is dense in $H^{1}$, a function $u \in H^{1}$ that satisfies the equation (3.4) solves therefore also the eigenvalue equation (2.6). If the function $u \in H^{1}$ solves conversely the equation $(2.6)$, for all functions $v \in \mathscr{D}$

$$
0=a(u, G v)-\lambda(u, G v)=\left(u+G V u, G^{-1} G v\right)=(u+G V u, v) .
$$

As $\mathscr{D}$ is dense in $L_{2}, u$ solves therefore the equation (3.4). Since the multiplication operator $V$ maps $H^{1}$ into $L_{2}$ and the operator $G$ the space $L_{2}$ into $H^{2}$, this means at the same time that $u$ is contained in $H^{2}$.

In the next step, we split the potential part $V u$ into the sum of a smooth part $Q V u$, the convolution $K * V u$ of $V u$ with a Gaussian kernel

$$
K(x)=\left(\frac{1}{4 \pi \gamma}\right)^{3 N / 2} \exp \left(-\frac{1}{4 \gamma}|x|^{2}\right)
$$

of a width that is determined by the constant $\gamma<1$ and will later be adapted to the needs, and the complementary part $P V u$. The equation (3.4), which is by Lemma 3.1 equivalent to the original Schrödinger equation (2.6), turns then into

$$
u+G P V u=-G Q V u \text {. }
$$

The operators $G, Q$, and $P=I-Q$ commute. This follows from the representation

$$
(Q f)(x)=\left(\frac{1}{\sqrt{2 \pi}}\right)^{3 N} \int \mathrm{e}^{-\gamma|\omega|^{2}} \widehat{f}(\omega) \mathrm{e}^{\mathrm{i} \omega \cdot x} \mathrm{~d} \omega
$$

of the convolution operator in terms of the Fourier transform. As the wavefunction $u$ under consideration satisfies the equation (3.4), therefore $G Q V u=-Q u$. Thus

$$
u+G P V u=Q u .
$$

The point is that, for sufficiently small $\gamma$, that is, a sufficiently small width of the smoothing kernel, the solution $u$ is completely determined by its regular part $Q u$. 
Lemma 3.2 For all square integrable functions $f$ and all $\gamma<1$,

$$
\|G P f\|_{1} \leq \sqrt{\gamma}\|f\|_{0} .
$$

Proof The proof is a rather immediate consequence of the Fourier representation

$$
\|G P f\|_{1}^{2}=\int\left(1+|\omega|^{2}\right)\left(\frac{1-\mathrm{e}^{-\gamma|\omega|^{2}}}{|\omega|^{2}-\lambda}\right)^{2}|\widehat{f}(\omega)|^{2} \mathrm{~d} \omega
$$

of the square of the norm to be estimated, of the elementary estimate

$$
(1+t)\left(\frac{1-\mathrm{e}^{-t}}{t}\right)^{2} \leq 1
$$

that holds for all $t>0$, and of Plancherel's theorem.

The $L_{2}$-norm of $V u$ can by (2.3) be estimated by the $H^{1}$-norm of $u$. The operator

$$
T: H^{1} \rightarrow H^{1}: u \rightarrow G P V u
$$

is thus for sufficiently small $\gamma$ contractive, more precisely, introducing the constant

$$
\theta(N, Z)=(2 Z+N-1) N^{1 / 2}
$$

already known from Lemma2.1, if the condition

$$
\sqrt{\gamma} \theta(N, Z)<1
$$

is satisfied. For any given $f \in H^{1}$, and in particular for $f=Q u$, the equation

$$
u+T u=f
$$

possesses then the uniquely determined solution

$$
u=(I+T)^{-1} f=\sum_{v=0}^{\infty}(-1)^{v} T^{v} f .
$$

This representation of the wavefunction $u$ represents the foundation of our theory.

\section{The influence of perturbations}

The basic idea is the approximation of the symbols of the operators of which the operator $T$ is composed with extremely high accuracy by series of Gauss functions. In this section we study how the solution of the equation (3.13) reacts to such perturbations of the operator $T$. Our starting point will be approximations

$$
\widetilde{V}: H^{1} \rightarrow L_{2}, \quad \widetilde{G}: L_{2} \rightarrow H^{2}
$$

of the multiplication operator $u \rightarrow V u$ and of the operator (3.1) for which

$$
\|V u-\widetilde{V} u\|_{0} \leq \theta(N, Z) \varepsilon|u|_{1}, \quad\|G f-\widetilde{G} f\|_{1} \leq \varepsilon\|G f\|_{1}
$$


holds for all $u \in H^{1}$ and $f \in L_{2}$, respectively, where $\varepsilon$ is a very small constant, by orders of magnitude less than the accuracy of the physical model and independent of the given eigenvalue. The difference of the perturbed operator

$$
\widetilde{T}=\widetilde{G} P \widetilde{V}
$$

and the operator 3.10 ) attains then the representation

$$
\widetilde{T}-T=(\widetilde{G}-G) P V+G P(\widetilde{V}-V)+(\widetilde{G}-G) P(\widetilde{V}-V),
$$

from which by assumption (4.2) at first the estimate

$$
\|\widetilde{T} u-T u\|_{1} \leq \varepsilon\|G P V u\|_{1}+(1+\varepsilon)\|G P(\widetilde{V} u-V u)\|_{1}
$$

follows. It implies by (3.9), Lemma 2.1, and (4.2) the estimate

$$
\|\widetilde{T} u-T u\|_{1} \leq \delta|u|_{1}, \quad \delta=\theta(N, Z) \gamma^{1 / 2}\left(2 \varepsilon+\varepsilon^{2}\right) .
$$

That is, the perturbed operator (4.3) is already for $\delta<1-\|T\|_{1}$ contractive and differs with the given $\delta$ only very little from $T$. The distance between our eigenfunction $u$, the solution of the original equation $(3.8)$ here rewritten as

$$
u+\widetilde{T} u=Q u+(\widetilde{T} u-T u)
$$

and the solution $\widetilde{u}$ of the modified equation

$$
\widetilde{u}+\widetilde{T} \widetilde{u}=Q u
$$

in which the term $\widetilde{T} u-T u=P(\widetilde{G} \widetilde{V} u-G V u)$ is neglected, satisfies then the estimate

$$
\|u-\widetilde{u}\|_{1} \leq \frac{\delta}{1-\|\widetilde{T}\|_{1}}|u|_{1} .
$$

The accuracy of the approximation of the operators thus transfers almost completely to the solution $\tilde{u}$ of the perturbed equation.

The smooth part $Q u$ of the eigenfunction $u$, its convolution with the kernel (3.5), reflects the global structure of $u$. The level of resolution is determined by the width of the kernel. The transition to the solution $\tilde{u}$ of the perturbed equation (4.8) adds the missing information on the singularities of $u$ up to a many orders of magnitude higher level of resolution and boosts the accuracy correspondingly. If $\gamma<1$,

$$
\|u-Q u\|_{1} \leq \gamma^{1 / 2}|u|_{2},
$$

as is shown analogously to (3.9). This has to be related to the $H^{1}$-distance between the exact eigenfunction $u$ and its approximation $\widetilde{u}$, that behaves by (4.9) like

$$
\|u-\widetilde{u}\|_{1} \lesssim \gamma^{1 / 2} \varepsilon|u|_{1} .
$$

That is, one gains a factor of order $\varepsilon$, of the size of the approximation errors 4.2). This justifies to neglect the term $\widetilde{T} u-T u$ in equation (4.7). 
As stated in Sect. 2, the Fourier transform of the eigenfunction $u$ is real-analytic and all its derivatives are bounded. Like its Fourier transform, $f=Q u$ is thus a rapidly decreasing function and can therefore be expanded into a series of Gauss-Hermite functions or more precisely of eigenfunctions of the harmonic oscillator that converges in the $H^{1}$-norm super-algebraically. Our goal is to show that, with a corresponding choice of the approximations 4.1) of $G$ and $V$, the solution

$$
\widetilde{u}=\sum_{v=0}^{\infty}(-1)^{v} \widetilde{T}^{v} f
$$

of the equation (4.8) can be approximated up to given accuracy with a comparable number of Gauss functions as $f$, provided the width of the smoothing kernel is chosen sufficiently small in dependence of the approximation order aimed for.

\section{The core of the approximation process}

The key to our approximation of the potential $V$ and of the inverse $G$ of the shifted Laplace operator is the approximation of the functions $1 / r^{\beta}, \beta=1 / 2$ and 1 , with very high relative accuracy by infinite series of exponential functions. One obtains such expansions discretizing representations of $1 / r^{\beta}$ in form of integrals over the real axis. We will work with a particular such construction due to Beylkin and Monzón [4]. Their starting point is a representation of the gamma function

$$
\Gamma(z)=\int_{0}^{\infty} \mathrm{e}^{-t} t^{z-1} \mathrm{~d} t, \quad \operatorname{Re} z>0
$$

in terms of an integral of a rapidly decreasing function.

Lemma 5.1 For all real $r>0$ and all complex $z$ with positive real part,

$$
\Gamma(z)=r^{z} \int_{-\infty}^{\infty} \exp \left(-r \mathrm{e}^{t}+z t\right) \mathrm{d} t
$$

Proof The substitution $\varphi(t)=r \mathrm{e}^{t}$ yields

$$
\Gamma(z)=\int_{-\infty}^{\infty} \mathrm{e}^{-\varphi(t)} \varphi(t)^{z-1} \varphi^{\prime}(t) \mathrm{d} t
$$

Written out, this is the representation (5.2).

The representation (5.2) of $\Gamma(z)$ leads conversely to the representation

$$
\frac{1}{r^{\beta}}=\frac{1}{\Gamma(\beta)} \int_{-\infty}^{\infty} \exp \left(-r \mathrm{e}^{t}+\beta t\right) \mathrm{d} t
$$

of the function $r \rightarrow 1 / r^{\beta}, r>0$, for arbitrary exponents $\beta>0$. The integrand decays for $t \rightarrow-\infty$ like $\mathrm{e}^{\beta t}$, and for $t \rightarrow \infty$ even more rapidly. The idea is to discretize this integral with the trapezoidal rule. This yields the approximation

$$
\frac{1}{r^{\beta}} \approx \frac{1}{\Gamma(\beta)} h \sum_{k=-\infty}^{\infty} \mathrm{e}^{\beta k h} \exp \left(-\mathrm{e}^{k h} r\right)=\frac{1}{r^{\beta}} \phi(\ln r)
$$


that depends only on the distance $h$ of the quadrature points. The function

$$
\phi(s)=\frac{1}{\Gamma(\beta)} h \sum_{k=-\infty}^{\infty} \exp \left(-\mathrm{e}^{k h+s}+\beta(k h+s)\right)
$$

is continuous due to the uniform convergence of the series on bounded intervals and periodic with period $h$ by definition. The relative error is therefore uniformly bounded in $r$ and attains its maximum on every interval $\mathrm{e}^{s} \leq r \leq \mathrm{e}^{s+h}$. The high accuracy of the approximation is a consequence of the following observation.

Lemma 5.2 The function (5.5) possesses a series representation

$$
\phi(s)=1+\frac{2}{\Gamma(\beta)} \sum_{\ell=1}^{\infty}\left|\Gamma\left(\beta-\mathrm{i} \frac{2 \pi \ell}{h}\right)\right| \sin \left(\frac{2 \pi \ell}{h}\left(s-s_{\ell}\right)\right),
$$

with certain phase shifts $s_{\ell}$.

Proof The proof is based on the Poisson summation formula for rapidly decreasing functions $f: \mathbb{R} \rightarrow \mathbb{R}$, more precisely on its rescaled variant

$$
h \sum_{k=-\infty}^{\infty} f(k h)=\sqrt{2 \pi} \sum_{\ell=-\infty}^{\infty} \widehat{f}\left(\frac{2 \pi \ell}{h}\right) .
$$

The Poisson summation formula is applied to the function

$$
f(t)=\exp \left(-\mathrm{e}^{t+s}+\beta(t+s)\right)
$$

whose Fourier transform can be calculated with help of the representation of the gamma function from Lemma5.1 This leads to the complex representation

$$
\phi(s)=\frac{1}{\Gamma(\beta)} \sum_{\ell=-\infty}^{\infty} \Gamma\left(\beta-\mathrm{i} \frac{2 \pi \ell}{h}\right) \exp \left(\mathrm{i} \frac{2 \pi \ell}{h} s\right)
$$

of the function (5.5). As $\Gamma(\beta+\mathrm{i} \omega)$ is the complex conjugate of $\Gamma(\beta-\mathrm{i} \omega)$, the right-hand side is real-valued and can be written as above.

It will turn out shortly that at least for $\beta=1 / 2$ and $\beta=1$, and not too big values of $h$, the term $\ell=1$ completely dominates the series; the other terms sum up to a remainder whose maximum norm is many orders of magnitude smaller than that of this term. A direct consequence of Lemma 5.2 is the following error estimate.

Lemma 5.3 For all positive real exponents $\beta$ and all $r>0$,

$$
\left|\frac{1}{r^{\beta}}-\frac{1}{\Gamma(\beta)} h \sum_{k=-\infty}^{\infty} \mathrm{e}^{\beta k h} \exp \left(-\mathrm{e}^{k h} r\right)\right| \leq \frac{\varepsilon(\beta, h)}{r^{\beta}}
$$

holds, where the bound $\varepsilon(\beta, h)$ for the relative error is given by the expression

$$
\varepsilon(\beta, h)=\frac{2}{\Gamma(\beta)} \sum_{\ell=1}^{\infty}\left|\Gamma\left(\beta-\mathrm{i} \frac{2 \pi \ell}{h}\right)\right| .
$$


Beylkin and Monzón [4] estimate the error bound $\varepsilon(\beta, h)$ for arbitrary positive real exponents $\beta$ and show that it tends exponentially to zero as $h$ goes to zero. We are solely interested in the exponents $\beta=1 / 2$ and $\beta=1$ and utilize that for real $\omega$

$$
\left|\Gamma\left(\frac{1}{2}-\mathrm{i} \omega\right)\right|^{2}=\frac{\pi}{\cosh (\pi \omega)}, \quad|\Gamma(1-\mathrm{i} \omega)|^{2}=\frac{\pi \omega}{\sinh (\pi \omega)}
$$

holds, which is a direct consequence of Euler's reflection formula for the gamma function. The error bound $\varepsilon(1 / 2, h)$ possesses therefore the series representation

$$
\varepsilon\left(\frac{1}{2}, h\right)=2 \sqrt{2} \sum_{\ell=1}^{\infty} \frac{q^{\ell}}{\left(1+q^{4 \ell}\right)^{1 / 2}}
$$

in terms of the variable $q=\mathrm{e}^{-\pi^{2} / h}$, and the error bound $\varepsilon(1, h)$ the expansion

$$
\varepsilon(1, h)=4 \pi h^{-1 / 2} \sum_{\ell=1}^{\infty} \frac{\sqrt{\ell} q^{\ell}}{\left(1-q^{4 \ell}\right)^{1 / 2}} .
$$

We conclude that the maximum norm of the relative error itself as well as its upper bound $\varepsilon(\beta, h)$ differ for $\beta=1 / 2$ and $\beta=1$, respectively, from the quantities

$$
2 \sqrt{2} \mathrm{e}^{-\pi^{2} / h}, \quad 4 \pi h^{-1 / 2} \mathrm{e}^{-\pi^{2} / h}
$$

only by factors that tend themselves like $\sim \mathrm{e}^{-\pi^{2} / h}$ to one as $h$ goes to zero. The relative error thus tends very rapidly to zero. For both cases, it is already less than $10^{-7}$ for $h=1 / 2$, less than $10^{-15}$ for $h=1 / 4$, and less than $10^{-32}$ for $h=1 / 8$.

\section{The approximations of the operators}

The approximations (5.4) of $1 / \sqrt{r}$ and $1 / r$ lead to the approximations

$$
\frac{1}{r} \approx \frac{1}{\sqrt{\pi}} h \sum_{k=-\infty}^{\infty} \mathrm{e}^{k h / 2} \exp \left(-\mathrm{e}^{k h} r^{2}\right), \quad \frac{1}{r} \approx h \sum_{k=-\infty}^{\infty} \mathrm{e}^{k h} \exp \left(-\mathrm{e}^{k h} r\right)
$$

of $1 / r$ by sums of Gauss and exponential functions, respectively. These form the basis of our approximations (4.1) of the interaction potentials between the electrons and the electrons and nuclei and of the inverse 3.1):

$$
(G f)(x)=\left(\frac{1}{\sqrt{2 \pi}}\right)^{3 N} \int \frac{1}{|\omega|^{2}-\lambda} \widehat{f}(\omega) \mathrm{e}^{\mathrm{i} \omega \cdot x} \mathrm{~d} \omega
$$

of the shifted Laplace operator $-\Delta-\lambda$. It is approximated replacing its symbol

$$
\frac{1}{|\omega|^{2}-\lambda} \approx h \sum_{k=-\infty}^{\infty} \mathrm{e}^{k h} \exp \left(-\mathrm{e}^{k h}\left(|\omega|^{2}-\lambda\right)\right)
$$

by the second of the approximations from 6.1). That is, we replace $G$ by the sum

$$
\widetilde{G}=\sum_{k=-\infty}^{\infty} G_{k}
$$


of the operators $G_{k}: L_{2} \rightarrow H^{2}$ with symbols

$$
h \exp \left(\mathrm{e}^{k h} \lambda+k h\right) \exp \left(-\mathrm{e}^{k h}|\omega|^{2}\right) .
$$

The pointwise convergence of the series (6.4) to its limit $\widetilde{G}: L_{2} \rightarrow H^{2}$ can be shown using 5.7 and the dominated convergence theorem. Its norm convergence with respect to an appropriate scale of norms will follow from the considerations in the next section. For square integrable functions $f$

$$
\|G f-\widetilde{G} f\|_{1} \leq \varepsilon(1, h)\|G f\|_{1}
$$

holds, as follows from Lemma 5.3. The interaction potential

$$
V(x)=-\sum_{i=1}^{N} \sum_{v=1}^{K} \frac{Z_{v}}{\left|x_{i}-a_{v}\right|}+\frac{1}{2} \sum_{\substack{i, j=1 \\ i \neq j}}^{N} \frac{1}{\left|x_{i}-x_{j}\right|}
$$

is treated correspondingly replacing it by the sum

$$
\widetilde{V}=\sum_{k=-\infty}^{\infty} V_{k}
$$

of the parts given by the expression

$$
V_{k}(x)=-\sum_{i=1}^{N} \sum_{v=1}^{K} Z_{v} \phi_{k}\left(x_{i}-a_{v}\right)+\frac{1}{2} \sum_{\substack{i, j=1 \\ i \neq j}}^{N} \phi_{k}\left(x_{i}-x_{j}\right),
$$

where the $\phi_{k}: \mathbb{R}^{3} \rightarrow \mathbb{R}$ are the Gauss functions

$$
\phi_{k}(x)=\frac{1}{\sqrt{\pi}} h \mathrm{e}^{k h / 2} \exp \left(-\mathrm{e}^{k h}|x|^{2}\right) .
$$

The approximation error for the single interaction terms can again be estimated with the help of the estimate (5.7) from Lemma 5.3. The three-dimensional Hardy inequality (2.4) and some elementary calculations using the triangle and the Cauchy-Schwarz inequality lead to the error estimate

$$
\|V u-\widetilde{V} u\|_{0} \leq \theta(N, Z) \varepsilon(1 / 2, h)|u|_{1}
$$

for functions $u \in H^{1}$, where the prefactor (3.11):

$$
\theta(N, Z)=(2 Z+N-1) N^{1 / 2}
$$

is that from Lemma 2.1 and $Z$ is the total charge of the nuclei. We conclude that already for a moderate choice of the distance $h$ between the quadrature points in (5.4) and (6.1), respectively, we get approximations of extremely high accuracy of the inverse $G$ of the shifted Laplace operator and of the multiplication operator $V$. The choice of the gridsize $h$ fixes the substitute problem (4.8) that replaces the original equation (3.8) and (4.7), respectively, and whose solution we want to approximate in what follows instead of the given eigenfunction. 
The crucial point is that the single parts of which the approximate operators are composed map Gauss functions to Gauss functions, pure Gauss functions to pure Gauss functions and Gauss-Hermite functions to Gauss-Hermite functions with a polynomial part of same degree. Let us discuss at first the case of pure Gauss function, without polynomial part. The multiplication of such a Gauss function with one of the parts $V_{k}$ yields a finite linear combination of such Gauss functions. The reason is that the product of two such Gauss functions

$$
\exp \left(-\frac{1}{2}\left(x-a_{1}\right) \cdot Q_{1}\left(x-a_{1}\right)\right), \quad \exp \left(-\frac{1}{2}\left(x-a_{2}\right) \cdot Q_{2}\left(x-a_{2}\right)\right)
$$

is a scalar multiple of the Gauss function

$$
\exp \left(-\frac{1}{2}(x-a) \cdot Q(x-a)\right)
$$

with matrix and shift

$$
Q=Q_{1}+Q_{2}, \quad a=\left(Q_{1}+Q_{2}\right)^{-1}\left(Q_{1} a_{1}+Q_{2} a_{2}\right) .
$$

Or consider the application of one of the operators $G_{k}$ or of the operator (3.7) to a Gauss function (6.14). The Fourier transform of this Gauss function is

$$
\frac{1}{\sqrt{\operatorname{det} Q}} \mathrm{e}^{-\mathrm{i} a \cdot \omega} \exp \left(-\frac{1}{2} \omega \cdot Q^{-1} \omega\right) \text {. }
$$

Multiplication with an isotropic Gauss function

$$
\exp \left(-\frac{1}{2} \alpha|\omega|^{2}\right), \quad \alpha>0
$$

and application of the inverse Fourier transform lead to the Gauss function

$$
\frac{1}{\sqrt{\operatorname{det}(I+\alpha Q)}} \exp \left(-\frac{1}{2}(x-a) \cdot(I+\alpha Q)^{-1} Q(x-a)\right) \text {. }
$$

If the matrices under consideration are of the form $Q=Q^{\prime} \otimes I_{3}$, with symmetricpositive definite matrices $Q^{\prime}$ of dimension $N$ and the $(3 \times 3)$-identity matrix $I_{3}$, none of the operations leads out of this class, so that all computations can be reduced to computations in the $\mathrm{N}$-dimensional space.

The argumentation for Gauss-Hermite functions is similar. This is obvious for the multiplication with one of the parts $V_{k}$ because this does not affect the polynomial factor. The rest follows from the fact that there is a direct correspondence between the multiplication of a function with a polynomial and the derivatives of its Fourier transform. For all multi-indices $\alpha$ and all rapidly decreasing functions $v$,

$$
\mathscr{F}\left(x^{\alpha} v\right)=\mathrm{i}^{|\alpha|} \mathrm{D}^{\alpha} \mathscr{F} v, \quad \mathscr{F}^{-1}\left(\omega^{\alpha} v\right)=(-\mathrm{i})^{|\alpha|} \mathrm{D}^{\alpha} \mathscr{F}^{-1} v
$$

If $v$ is a Gauss function (now without polynomial part) centered around the origin, the derivatives $\mathrm{D}^{\alpha} \mathscr{F} v$ and $\mathrm{D}^{\alpha} \mathscr{F}^{-1} v$ of the Fourier transform and of the inverse Fourier transform of $v$ are products of the Gauss functions $\mathscr{F} v$ and $\mathscr{F}^{-1} v$ with polynomials 
of degree $|\alpha|$, so that one remains in the given class. A shift of the center is easily included and does not affect this property. The hope is therefore that the solution of our substitute equation, the function

$$
\widetilde{u}=\sum_{v=0}^{\infty}(-1)^{v} \widetilde{T}^{v} f
$$

can be well approximated by linear combinations of Gauss functions as long as this holds for the right hand side $f$ of this equation.

\section{Estimates of the norms of the single components}

To obtain approximations of the solution (4.12), 6.20) of the substitute equation (4.8) by finite linear combinations of Gauss functions, we need to truncate both the series on the right hand side of 6.20) itself and the series of Gauss functions representing the single terms $\widetilde{T}^{v} f$. For this purpose we utilize estimates in fractional order Sobolev spaces $H^{\vartheta}$ for the norms of the single parts of which the approximate operators 6.4 and (6.8) are composed. We will show in this section that the norms of these single parts tend exponentially to zero as $k$ goes to plus or minus infinity.

The Sobolev space $H^{\vartheta}, \vartheta$ an arbitrary real number, is the completion of the space of the real-valued, rapidly decreasing functions, or even the infinitely differentiable functions with compact support, under the norm given by the expression

$$
\|u\|_{\vartheta}^{2}=\int\left(1+|\omega|^{2}\right)^{\vartheta}|\widehat{u}(\omega)|^{2} \mathrm{~d} \omega .
$$

For $\vartheta=0$, this is the $L_{2}$-norm and the corresponding Sobolev space $H^{\vartheta}$ is $L_{2}$, and for $\vartheta=1$, the norm coincides with the standard norm on $H^{1}$. If $\vartheta$ is an integer greater than zero, $H^{\vartheta}$ consists of the $\vartheta$-times weakly differentiable functions with weak derivatives in $L_{2}$. For values $\vartheta>0$, we use also the seminorm on $H^{\vartheta}$ given by

$$
|u|_{\vartheta}^{2}=\int|\omega|^{2 \vartheta}|\widehat{u}(\omega)|^{2} \mathrm{~d} \omega
$$

For $\vartheta=1$, this seminorm is again the usual seminorm on $H^{1}$.

We begin with the parts of which the interaction potentials are composed. Starting point is the estimate from the next lemma for functions $u: \mathbb{R}^{3} \rightarrow \mathbb{R}$ that is based on the three-dimensional Hardy-Rellich inequality. For $u \in H^{\vartheta}, 0<\vartheta<3 / 2$,

$$
\int \frac{1}{|x|^{2 \vartheta}}|u(x)|^{2} \mathrm{~d} x \leq \frac{4^{\vartheta}}{\min \left(1,(3-2 \vartheta)^{2}\right)} \int|\omega|^{2 \vartheta}|\widehat{u}(\omega)|^{2} \mathrm{~d} \omega .
$$

For $\vartheta=1$, this is the classical Hardy inequality (2.4). For $\vartheta<1$, the inequality can be derived by interpolation from the classical Hardy inequality. For $1<\vartheta<3 / 2$, the inequality is at first reduced, similarly as in the proof from [20] of the classical Hardy inequality, to an estimate of the given weighted norm of $u$ by a weighted norm of $\nabla u$ that is then further estimated using the inequality for the already known case $\vartheta<1$. The optimal constant can be calculated with much more effort expanding the 
functions under consideration into products of radial parts and spherical harmonics; see [18]. It behaves like $\sim 1 /(3-2 \vartheta)^{2}$ when $\vartheta$ approaches the limit value $3 / 2$, a behavior that reflects the estimate (7.3) properly.

Lemma 7.1 For all indices $0<\vartheta<1 / 2$, all nonnegative integers $k$, and all rapidly decreasing functions $u: \mathbb{R}^{3} \rightarrow \mathbb{R}$, the estimate

$$
\left\|\phi_{k} u\right\|_{0} \leq \kappa(\vartheta) \frac{\vartheta h}{2} \exp \left(-\frac{\vartheta h}{2}|k|\right)|u|_{1+\vartheta}
$$

holds, where the $\phi_{k}$ are given by (6.10) and the constant $\kappa(\vartheta)$ is defined by

$$
\kappa(\vartheta)=\frac{1}{\sqrt{\pi}}\left(\frac{2+2 \vartheta}{\mathrm{e}}\right)^{(1+\vartheta) / 2} \frac{2}{\vartheta(1-2 \vartheta)}
$$

For negative integers $k$, the following estimate holds:

$$
\left\|\phi_{k} u\right\|_{0} \leq \kappa(\vartheta) \frac{\vartheta h}{2} \exp \left(-\frac{\vartheta h}{2}|k|\right)|u|_{1-\vartheta}
$$

Proof The square of the $L_{2}$-norm to be estimated can be written as

$$
\left\|\phi_{k} u\right\|_{0}^{2}=\frac{h^{2}}{\pi} \mathrm{e}^{-\vartheta k h} \int\left(\mathrm{e}^{k h}|x|^{2}\right)^{1+\vartheta} \exp \left(-2 \mathrm{e}^{k h}|x|^{2}\right) \frac{1}{|x|^{2(1+\vartheta)}}|u(x)|^{2} \mathrm{~d} x .
$$

Since the expression $t^{1+\vartheta} \mathrm{e}^{-2 t}, t \geq 0$, attains its maximum at $t=(1+\vartheta) / 2$,

$$
\left\|\phi_{k} u\right\|_{0}^{2} \leq \frac{h^{2}}{\pi}\left(\frac{1+\vartheta}{2 \mathrm{e}}\right)^{1+\vartheta} \mathrm{e}^{-\vartheta k h} \int \frac{1}{|x|^{2(1+\vartheta)}}|u(x)|^{2} \mathrm{~d} x
$$

follows. The Hardy inequality (7.3) yields

$$
\left\|\phi_{k} u\right\|_{0}^{2} \leq \frac{h^{2}}{\pi}\left(\frac{1+\vartheta}{2 \mathrm{e}}\right)^{1+\vartheta} \frac{4^{1+\vartheta}}{(1-2 \vartheta)^{2}} \mathrm{e}^{-\vartheta k h}|u|_{1+\vartheta}^{2}
$$

For $k \geq 0$, this is the estimate (7.4). For $k<0$, one starts from the representation

$$
\left\|\phi_{k} u\right\|_{0}^{2}=\frac{h^{2}}{\pi} \mathrm{e}^{\vartheta k h} \int\left(\mathrm{e}^{k h}|x|^{2}\right)^{1-\vartheta} \exp \left(-2 \mathrm{e}^{k h}|x|^{2}\right) \frac{1}{|x|^{2(1-\vartheta)}}|u(x)|^{2} \mathrm{~d} x
$$

and obtains correspondingly the estimate

$$
\left\|\phi_{k} u\right\|_{0}^{2} \leq \frac{h^{2}}{\pi}\left(\frac{1-\vartheta}{2 \mathrm{e}}\right)^{1-\vartheta} 4^{1-\vartheta} \mathrm{e}^{\vartheta k h}|u|_{1-\vartheta}^{2}
$$

Since for all $\vartheta$ in the interval under consideration

$$
\left(\frac{2-2 \vartheta}{\mathrm{e}}\right)^{1-\vartheta} \leq\left(\frac{2+2 \vartheta}{\mathrm{e}}\right)^{1+\vartheta} \frac{1}{(1-2 \vartheta)^{2}}
$$

holds, this proves the estimate (7.6) for the case of negative integers $k$. 
Next we transfer these estimates to the multi-particle case and consider functions

$$
u:\left(\mathbb{R}^{3}\right)^{N} \rightarrow \mathbb{R}:\left(x_{1}, \ldots, x_{N}\right) \rightarrow u\left(x_{1}, \ldots, x_{N}\right) .
$$

Lemma 7.2 For $0<\vartheta<1 / 2$, for all nonnegative integers $k$, for all indices $i \neq j$, and for all rapidly decreasing functions $u: \mathbb{R}^{3 N} \rightarrow \mathbb{R}$,

$$
\int\left|\phi_{k}\left(x_{i}-x_{j}\right) u(x)\right|^{2} \mathrm{~d} x \leq K^{2} \int\left|\omega_{i}\right|^{2(1+\vartheta)}|\widehat{u}(\omega)|^{2} \mathrm{~d} \omega,
$$

where $K$ is here an abbreviation for the expression

$$
K=\kappa(\vartheta) \frac{\vartheta h}{2} \exp \left(-\frac{\vartheta h}{2}|k|\right) .
$$

For negative integers $k$, the following estimate holds:

$$
\int\left|\phi_{k}\left(x_{i}-x_{j}\right) u(x)\right|^{2} \mathrm{~d} x \leq K^{2} \int\left|\omega_{i}\right|^{2(1-\vartheta)}|\widehat{u}(\omega)|^{2} \mathrm{~d} \omega .
$$

Proof We split the vectors $x=\left(x_{i}, x^{\prime}\right)$ in $\mathbb{R}^{3 N}$ into the component $x_{i}$ in $\mathbb{R}^{3}$ and the remaining part $x^{\prime}$. As, for any given $x^{\prime}, x_{i} \rightarrow u\left(x_{i}, x^{\prime}\right)$ is a rapidly decreasing function from $\mathbb{R}^{3}$ to $\mathbb{R}$ and as the seminorms $(7.2)$ are shift-invariant, by Lemma 7.1 then

$$
\int\left|\phi_{k}\left(x_{i}-x_{j}\right) u\left(x_{i}, x^{\prime}\right)\right|^{2} \mathrm{~d} x_{i} \leq K^{2} \int\left|\omega_{i}\right|^{2(1+\vartheta)}\left|\left(\mathscr{F}_{i} u\right)\left(\omega_{i}, x^{\prime}\right)\right|^{2} \mathrm{~d} \omega_{i}
$$

where $\mathscr{F}_{i}$ denotes the Fourier transform with respect to $x_{i}$. Correspondingly, let $\mathscr{F}^{\prime}$ be the Fourier transform with respect to the remaining variables $x^{\prime}$. Integration of this inequality with respect to the remaining variables, Fubini's theorem, that

$$
\int\left|\left(\mathscr{F}_{i} u\right)\left(\omega_{i}, x^{\prime}\right)\right|^{2} \mathrm{~d} x^{\prime}=\int\left|\left(\mathscr{F}^{\prime} \mathscr{F}_{i} u\right)\left(\omega_{i}, \omega^{\prime}\right)\right|^{2} \mathrm{~d} \omega^{\prime}
$$

by Plancherel's theorem, and the observation that $\mathscr{F}^{\prime} \mathscr{F}_{i}$ is the Fourier transform with respect to the full set of variables, finally yield the estimate (7.8). The estimate (7.10) for the case of negative integers $k$ is proved in the same way.

Estimates of the same type hold for the terms coming from the interaction between the electrons and the nuclei and can be derived in the same way.

Lemma 7.3 For $0<\vartheta<1 / 2$, for all nonnegative integers $k$, for all indices $i$ and $v$, and for all rapidly decreasing functions $u: \mathbb{R}^{3 N} \rightarrow \mathbb{R}$,

$$
\int\left|\phi_{k}\left(x_{i}-a_{v}\right) u(x)\right|^{2} \mathrm{~d} x \leq K^{2} \int\left|\omega_{i}\right|^{2(1+\vartheta)}|\widehat{u}(\omega)|^{2} \mathrm{~d} \omega,
$$

where $K$ is again the abbreviation for the expression

$$
K=\kappa(\vartheta) \frac{\vartheta h}{2} \exp \left(-\frac{\vartheta h}{2}|k|\right) .
$$

For negative integers $k$, the following estimate holds:

$$
\int\left|\phi_{k}\left(x_{i}-a_{v}\right) u(x)\right|^{2} \mathrm{~d} x \leq K^{2} \int\left|\omega_{i}\right|^{2(1-\vartheta)}|\widehat{u}(\omega)|^{2} \mathrm{~d} \omega .
$$


We finally combine the estimates from Lemma 7.2 and Lemma 7.3 for its parts to estimates for the functions 6.9), interpreted as a multiplication operators mapping the functions in the space $H^{1+\vartheta}$ to functions in $L_{2}$.

Lemma 7.4 For all indices $0<\vartheta<1 / 2$, for all negative integers $k$, and for all rapidly decreasing functions $u: \mathbb{R}^{3 N} \rightarrow \mathbb{R}$,

$$
\left\|V_{k} u\right\|_{0} \leq \frac{(2 Z+N-1) N^{(1+\vartheta) / 2}}{2} \kappa(\vartheta) \frac{\vartheta h}{2} \exp \left(-\frac{\vartheta h}{2}|k|\right)|u|_{1-\vartheta} .
$$

For nonnegative integers $k$, the following estimate holds:

$$
\left\|V_{k} u\right\|_{0} \leq \frac{(2 Z+N-1) N^{1 / 2}}{2} \kappa(\vartheta) \frac{\vartheta h}{2} \exp \left(-\frac{\vartheta h}{2}|k|\right)|u|_{1+\vartheta}
$$

As before, $Z=\sum_{v} Z_{v}$ is here again the total charge of the nuclei.

Proof For negative integers $k$, the estimates (7.10) and (7.13) for the single parts of $V_{k}$ and the triangle and the Cauchy-Schwarz inequality at first yield

$$
\left\|V_{k} u\right\|_{0} \leq \frac{(2 Z+N-1) N^{1 / 2}}{2}\left(K^{2} \sum_{i=1}^{N} \int\left|\omega_{i}\right|^{2(1-\vartheta)}|\widehat{u}(\omega)|^{2} \mathrm{~d} \omega\right)^{1 / 2},
$$

where the abbreviation 7.9 has again been used. By Hölder's inequality

$$
\sum_{i=1}^{N} \eta_{i}^{2(1-\vartheta)} \leq N^{\vartheta}
$$

for all $\eta$ on the surface of the $N$-dimensional unit sphere, from which 7.14 follows. The case of nonnegative integers $k$ is treated analogously, where the estimate

$$
\sum_{i=1}^{N} \eta_{i}^{2(1+\vartheta)} \leq 1
$$

for the $\eta$ on the surface of the $N$-dimensional unit sphere enters.

Because of $|u|_{1 \pm \vartheta} \leq\|u\|_{1+\vartheta}$, the two estimates can be combined to the estimate

$$
\left\|V_{k} u\right\|_{0} \leq \frac{(2 Z+N-1) N^{(1+\vartheta) / 2}}{2} \kappa(\vartheta) \frac{\vartheta h}{2} \exp \left(-\frac{\vartheta h}{2}|k|\right)\|u\|_{1+\vartheta}
$$

that holds both for positive and negative integers $k$.

Next we estimate the norms of the operators $G_{k}$ of which the approximate inverse 6.4) of the shifted Laplacian $-\Delta-\lambda$ is composed. We interpret these operators as operators from $L_{2}$ to the spaces $H^{2-\vartheta}$ for indices $\vartheta$ between 0 and 2 .

Lemma 7.5 For all indices $0<\vartheta<2$, for all integers $k$, and for all rapidly decreasing functions $f: \mathbb{R}^{3 N} \rightarrow \mathbb{R}$ the following estimate holds:

$$
\left|G_{k} f\right|_{2-\vartheta} \leq h\left(\frac{2-\vartheta}{2 \mathrm{e}}\right)^{(2-\vartheta) / 2} \exp \left(\mathrm{e}^{k h} \lambda+\frac{\vartheta}{2} k h\right)\|f\|_{0} .
$$


Proof We rewrite the square of the left hand side of (7.17) at first in the form

$$
\left|G_{k} f\right|_{2-\vartheta}^{2}=h^{2} \exp \left(2 \mathrm{e}^{k h} \lambda+\vartheta k h\right) \int\left(\mathrm{e}^{k h}|\omega|^{2}\right)^{2-\vartheta} \exp \left(-2 \mathrm{e}^{k h}|\omega|^{2}\right)|\widehat{f}(\omega)|^{2} \mathrm{~d} \omega .
$$

Using that the expression $t^{2-\vartheta} \mathrm{e}^{-2 t}, t>0$, attains its maximum at $t=(2-\vartheta) / 2$,

$$
\left|G_{k} f\right|_{2-\vartheta}^{2} \leq h^{2}\left(\frac{2-\vartheta}{2 \mathrm{e}}\right)^{2-\vartheta} \exp \left(2 \mathrm{e}^{k h} \lambda+\vartheta k h\right) \int|\widehat{f}(\omega)|^{2} \mathrm{~d} \omega
$$

follows, which was the proposition.

The conclusion is that, for $0<\vartheta<2$, the norms of the operators

$$
G_{k}: L_{2} \rightarrow H^{2-\vartheta}
$$

tend again rapidly to zero as $k$ goes to plus or minus infinity, exponentially as $k$ goes to minus infinity, and super exponentially as $k$ goes to plus infinity. Because of

$$
\exp \left(\mathrm{e}^{t} \lambda+\frac{\vartheta}{2} t\right) \leq \max \left(1,\left(-\frac{\vartheta}{\lambda \mathrm{e}}\right)^{\vartheta}\right) \exp \left(-\frac{\vartheta}{2}|t|\right)
$$

one obtains again, with the correspondingly chosen constant $\kappa^{*}(\lambda, \vartheta)$, the in comparison to the original estimate (7.17) for later purposes more convenient estimate

$$
\left|G_{k} f\right|_{2-\vartheta} \leq \kappa^{*}(\lambda, \vartheta) \frac{\vartheta h}{2} \exp \left(-\frac{\vartheta h}{2}|k|\right)\|f\|_{0}
$$

for $f \in L_{2}$, which, however, severely overestimates the norms for positive $k$.

Central for our argumentation is the splitting of the potential term $V u$ into the smooth part $Q V u$, the convolution of $V u$ with the Gaussian kernel (3.5), and the complementary part $P V u$. The cut-off operator $P$ filtering out the low-frequency part of $V u$ will make sure that the norms of the combined operators remain sufficiently small and do not exceed certain bounds. It reads in terms of the Fourier transform

$$
\widehat{P v}(\omega)=\left(1-\mathrm{e}^{-\gamma|\omega|^{2}}\right) \widehat{v}(\omega) .
$$

The constant $\gamma<1$ determines how strongly low frequencies are damped and how much $P$ reduces norms in the sense of the following estimate.

Lemma 7.6 For all indices $0<\vartheta<1 / 2$, all constants $\gamma<1$, and all rapidly decreasing functions $v: \mathbb{R}^{3 N} \rightarrow \mathbb{R}$, the following estimate holds:

$$
\|P v\|_{1+\vartheta} \leq \sqrt{2} \gamma^{1 / 2-\vartheta}|v|_{2-\vartheta} .
$$

Proof The square of the norm of $P v$ can be written as

$$
\|P v\|_{1+\vartheta}^{2}=\gamma^{1-2 \vartheta} \int a\left(\vartheta, \gamma, \gamma|\omega|^{2}\right)|\omega|^{2(2-\vartheta)}|\widehat{v}(\omega)|^{2} \mathrm{~d} \omega,
$$

where the function $a(\vartheta, \gamma, t)$ is given by

$$
a(\vartheta, \gamma, t)=t^{\vartheta}(\gamma+t)^{1+\vartheta}\left(\frac{1-\mathrm{e}^{-t}}{t}\right)^{2}
$$


and can for the given $\gamma$ and $\vartheta$ be roughly estimated as follows:

$$
a(\vartheta, \gamma, t) \leq(1+t)^{2}\left(\frac{1-\mathrm{e}^{-t}}{t}\right)^{2}
$$

To estimate the bound on the right hand side further, let

$$
f(t)=\mu t-(1+t)\left(1-\mathrm{e}^{-t}\right), \quad \mu=1+\mathrm{e}^{-1} .
$$

Since $f^{\prime \prime}(t)=-(1-t) \mathrm{e}^{-t}$, the derivative of $f$ takes its minimum $f^{\prime}(1)=0$ at $t=1$. That is, $f(t)$ increases strictly and it is $f(t) \geq f(0)=0$ for all $t \geq 0$. Therefore

$$
a(\vartheta, \gamma, t) \leq \mu^{2}
$$

Because e $\geq 5 / 2, \mu^{2} \leq 2$ and the estimate (7.21) is proven.

We could stop our considerations here and could as in [17] continue with the given estimate (7.16) for the $V_{k}$, seen as operators from $H^{1+\vartheta}$ to $L_{2}$, and the estimate resulting from (7.19) and (7.21) for the operators $G_{k} P=P G_{k}$ from $L_{2}$ back to $H^{1+\vartheta}$. This would result in an analysis on the approximation of the wavefunctions in a space $H^{1+\vartheta}$ for some $\vartheta$ between 0 and $1 / 2$. Since we are, however, finally interested in the approximation properties in the energy space underlying the electronic Schrödinger equation, the Sobolev space $H^{1}$, we shift our estimates still downward by the chosen $\vartheta$ using a simple duality and interpolation argument.

Lemma 7.7 The multiplication operators $V_{k}$ can be uniquely extended to bounded linear operators from $H^{1}$ to the dual space $H^{-\vartheta}, 0<\vartheta<1 / 2$. For all $u \in H^{1}$,

$$
\left\|V_{k} u\right\|_{-\vartheta} \leq \frac{(2 Z+N-1) N^{(1+\vartheta) / 2}}{2} \kappa(\vartheta) \frac{\vartheta h}{2} \exp \left(-\frac{\vartheta h}{2}|k|\right)\|u\|_{1} .
$$

Proof It suffices to prove the estimate for rapidly decreasing functions $u$ as these are dense in the spaces under consideration. Because $V_{k}$ maps rapidly decreasing functions to rapidly decreasing functions, this considerably simplifies the argumentation. We start from the estimate (7.16, here written as

$$
\left\|V_{k} u\right\|_{0} \leq c\|u\|_{1+\vartheta} .
$$

It implies that for all rapidly decreasing functions $\varphi$

$$
\left(V_{k} u, \varphi\right)=\left(u, V_{k} \varphi\right) \leq c\|u\|_{0}\|\varphi\|_{1+\vartheta} .
$$

A rapidly decreasing function $\varphi$ is real-valued if and only if $\overline{\hat{\varphi}(\omega)}=\widehat{\varphi}(-\omega)$ holds. Inserting the thus real-valued rapidly decreasing function $\varphi$ with Fourier transform

$$
\widehat{\varphi}(\omega)=\left(1+|\omega|^{2}\right)^{-(1+\vartheta)} \widehat{v}(\omega), \quad v=V_{k} u
$$

this leads by means of Plancherel's theorem to the dual estimate

$$
\left\|V_{k} u\right\|_{-(1+\vartheta)} \leq c\|u\|_{0} .
$$

The estimate (7.22) and generally for $0 \leq s \leq 1+\vartheta$ and $t=s-(1+\vartheta)$ the estimate

$$
\left\|V_{k} u\right\|_{t} \leq c\|u\|_{s}
$$

follow from the original and the dual estimate by interpolation within the space of the rapidly decreasing functions; details can be found in the appendix. 
For the composed operators $G_{k} P$ we proceed in the same way.

Lemma 7.8 The operators $G_{k} P$ can be uniquely extended to bounded linear operators from the dual space $H^{-\vartheta}, 0<\vartheta<1 / 2$, to $H^{1}$. For all $f \in H^{-\vartheta}$,

$$
\left\|G_{k} P f\right\|_{1} \leq \sqrt{2} \kappa^{*}(\lambda, \vartheta) \frac{\vartheta h}{2} \exp \left(-\frac{\vartheta h}{2}|k|\right) \gamma^{1 / 2-\vartheta}\|f\|_{-\vartheta} .
$$

Proof We take again advantage of the fact that it suffices to prove the estimate for rapidly decreasing functions $f$ and that $G_{k} P$ maps rapidly decreasing functions to rapidly decreasing functions. The fact that the operators $G_{k}$ and $P$ commute, the estimate (7.21) for the norm of the cut-off operator $P$, and the estimate (7.19) for the norm of the $G_{k}$ lead at first to the estimate

$$
\left\|G_{k} P f\right\|_{1+\vartheta} \leq c\|f\|_{0},
$$

where $c$ denotes here the constant from (7.23). For all rapidly decreasing functions $\varphi$ by Plancherel's theorem and the Cauchy-Schwarz inequality therefore

$$
\left(G_{k} P f, \varphi\right)=\left(f, G_{k} P \varphi\right) \leq c\|f\|_{-(1+\vartheta)}\|\varphi\|_{0} .
$$

Inserting the rapidly decreasing function $\varphi=G_{k} P f$, this yields the dual estimate

$$
\left\|G_{k} P f\right\|_{0} \leq c\|f\|_{-(1+\vartheta)} .
$$

The estimate (7.23) and generally for $0 \leq s \leq 1+\vartheta$ and $t=s-(1+\vartheta)$ the estimate

$$
\left\|G_{k} P f\right\|_{s} \leq c\|f\|_{t}
$$

follow from these two estimates again by interpolation.

Finally we combine the estimates from the last two lemmata to an estimate of the norm of the composed operators $G_{\ell} P V_{k}$ from the solution space $H^{1}$ back into itself. Introducing for abbreviation the prefactor

$$
\alpha=\frac{\kappa^{*}(\lambda, \vartheta) \kappa(\vartheta)(\vartheta h)^{2}(2 Z+N-1) N^{(1+\vartheta) / 2}}{4 \sqrt{2}} \gamma^{1 / 2-\vartheta}
$$

and moreover the constant

$$
q=\exp \left(-\frac{\vartheta h}{2}\right)
$$

this estimate for the norm of the composed operators reads

$$
\left\|G_{\ell} P V_{k} u\right\|_{1} \leq \alpha q^{|k|+|\ell|}\|u\|_{1} .
$$

The decisive point is that for an appropriate choice of the parameter $\gamma$ fixing the splitting of the wavefunctions into the smooth and the singular part the constant $\alpha$ can be made arbitrarily small and pushed below every bound. The reason is that the operators $V_{k}$ map $H^{1}$ to $H^{-\vartheta}$, but the $G_{\ell}$ conversely $H^{-\vartheta}$ to the space $H^{2-\vartheta}$ of higher 
regularity, which brings the cut-off operator $P$ into play. For ease of presentation, we still relabel the operators $G_{\ell} P V_{k}$ and denote them as

$$
T_{n, \ell}, \quad n=0,1,2, \ldots, \quad \ell=1, \ldots, \ell(n) .
$$

The index $n$ is associated with the exponential decay of their norms, such that

$$
\left\|T_{n, \ell} u\right\|_{1} \leq \alpha q^{n}\|u\|_{1}
$$

for $u \in H^{1}$. The index $\ell$ counts the operator products for which this estimate holds; there are $\ell(n)=\max (1,4 n)$ pairs of integers $k$ and $\ell$ for which $|k|+|\ell|=n$.

The aim of this work is to examine how well the solution $u$ of the equation

$$
u+\widetilde{T} u=f
$$

can be approximated by a linear combination of Gauss functions in terms of the corresponding approximation properties of the right hand side $f$. The two series

$$
\widetilde{V}=\sum_{k=-\infty}^{\infty} V_{k}, \quad \widetilde{G} P=\sum_{\ell=-\infty}^{\infty} G_{\ell} P
$$

of operators from $H^{1}$ to $H^{-\vartheta}$ and from $H^{-\vartheta}$ back to $H^{1}$ converge absolutely because of the exponential decay of the norms of the operators and the completeness of the corresponding spaces of linear operators. Their product

$$
\widetilde{T}=\left(\sum_{\ell=-\infty}^{\infty} G_{\ell} P\right)\left(\sum_{k=-\infty}^{\infty} V_{k}\right)
$$

can thus be written as Cauchy product, that is, in terms of the operators (7.27) as

$$
\widetilde{T}=\sum_{n=0}^{\infty} \sum_{\ell=1}^{\ell(n)} T_{n, \ell}
$$

As follows from the summation formula

$$
\sum_{n=0}^{\infty} \ell(n) q^{n}=1+4 \sum_{n=1}^{\infty} n q^{n}=\left(\frac{1+q}{1-q}\right)^{2}
$$

and the estimate (7.28) for the norms of the operators $T_{n, \ell}$,

$$
\sum_{n=0}^{\infty} \sum_{\ell=1}^{\ell(n)}\left\|T_{n, \ell}\right\|_{1} \leq \alpha\left(\frac{1+q}{1-q}\right)^{2} .
$$

The operator $\widetilde{T}$ from $H^{1}$ into itself is therefore a contraction for sufficiently small $\alpha$ and $\gamma$, respectively. The equation (7.29) possesses then for given $f \in H^{1}$ a unique solution $u \in H^{1}$. Note, however, that the condition on $\gamma$ resulting from (7.33) is more restrictive than the conditions discussed in Sect. 3 and Sect.4 


\section{The approximate solution of the substitute equation}

Our aim is to study how well the solution of the equation (7.29) can be approximated by a linear combination of Gauss functions in terms of corresponding approximation properties of the right hand side $f$. The solution possesses the representation

$$
u=\sum_{v=0}^{\infty}(-1)^{v} \widetilde{T}^{v} f
$$

In the first step of our analysis we study the approximability of the terms $\widetilde{T}^{v} f$.

Let $u \in H^{1}$ be a function that can be well approximated by low numbers of Gauss functions in the following sense. Assume that there is an infinite sequence $u_{1}, u_{2}, \ldots$ of Gauss functions such that, for every $\varepsilon>0$,

$$
\left\|u-\sum_{j=1}^{n(\varepsilon)} u_{j}\right\|_{1} \leq \varepsilon, \quad n(\varepsilon) \leq\left(\frac{\kappa}{\varepsilon}\right)^{1 / r}
$$

where $r>0$ is a given approximation order and $\kappa$ a constant that depends on $u$ but is independent of $\varepsilon$. If the integer $n(\varepsilon) \geq 0$ takes the value zero, the approximating sum is empty and the norm of $u$ itself already less than or equal to $\varepsilon$. We can assume that $n(\varepsilon)$ increases when $\varepsilon$ decreases; if necessary, one replaces $n(\varepsilon)$ simply by the minimum of all $n\left(\varepsilon^{\prime}\right)$ for $\varepsilon^{\prime} \leq \varepsilon$. Our first objective is to show that, with sufficiently small values of the constant $\alpha$ from (7.24), one can approximate the function

$$
\widetilde{T} u=\sum_{k=0}^{\infty} \sum_{\ell=1}^{\ell(k)} T_{k, \ell} u
$$

basically by half the number of Gauss functions with double accuracy.

These approximations are constructed as follows. First, we split the decay rate (7.25) in dependence of the order $r$ into the product $q=q_{1} q_{2}$ of two constants less than one chosen such that our later estimates become best possible. They are given by

$$
q_{1}=\exp \left(-\frac{1}{r+1} \frac{\vartheta h}{2}\right), \quad q_{2}=\exp \left(-\frac{r}{r+1} \frac{\vartheta h}{2}\right) .
$$

The approximations of $\widetilde{T} u$ are then the sums

$$
\sum_{k=0}^{\infty} \sum_{\ell=1}^{\ell(k)} \sum_{j=1}^{n_{k}} T_{k, \ell} u_{j}, \quad n_{k}=n\left(\delta^{-1} q_{2}^{-k} \varepsilon\right)
$$

of Gauss functions, where $\delta>0$ is here a new parameter that still needs to be fixed. These triple sums are finite because $n_{k}=0$ and the inner sums are thus empty as soon as the error bound $\delta^{-1} q_{2}^{-k} \varepsilon$ becomes greater than $\kappa$. The approximation error and the number of the remaining nonzero terms are estimated in next lemma. 
Lemma 8.1 Let $\varepsilon>0$ be arbitrary and choose the $n_{k}$ as in 8.5 . Then

$$
\left\|\sum_{k=0}^{\infty} \sum_{\ell=1}^{\ell(k)} T_{k, \ell} u-\sum_{k=0}^{\infty} \sum_{\ell=1}^{\ell(k)} \sum_{j=1}^{n_{k}} T_{k, \ell} u_{j}\right\|_{1} \leq \frac{\alpha}{\delta}\left(\frac{1+q_{1}}{1-q_{1}}\right)^{2} \varepsilon,
$$

where $\alpha$ is the constant from (7.24). Moreover,

$$
\sum_{k=0}^{\infty} \sum_{\ell=1}^{\ell(k)} n_{k} \leq \delta^{1 / r}\left(\frac{1+q_{1}}{1-q_{1}}\right)^{2}\left(\frac{\kappa}{\varepsilon}\right)^{1 / r}
$$

holds for the number of terms $T_{k, \ell} u_{j}$ of which the approximation (8.5) is composed.

Proof The left hand side of (8.6) can obviously be estimated by the double sum

$$
\sum_{k=0}^{\infty} \sum_{\ell=1}^{\ell(k)}\left\|T_{k, \ell}\left(u-\sum_{j=1}^{n_{k}} u_{j}\right)\right\|_{1}
$$

The error estimate 8.6 thus follows from $(7.28)$, the assumption $(8.2)$, that is,

$$
\left\|u-\sum_{j=1}^{n_{k}} u_{j}\right\|_{1} \leq \delta^{-1} q_{2}^{-k} \varepsilon
$$

in the present case, from $\left\|T_{k, \ell}\right\|_{1} \leq \alpha q^{k}, q q_{2}^{-1}=q_{1}, \ell(k)=\max (1,4 k)$, and

$$
\sum_{k=0}^{\infty} \ell(k) q_{1}^{k}=\left(\frac{1+q_{1}}{1-q_{1}}\right)^{2}
$$

Because $q_{2}^{1 / r}=q_{1}$, the estimate 8.7 for the number

$$
\sum_{k=0}^{\infty} \sum_{\ell=1}^{\ell(k)} n_{k} \leq \sum_{k=0}^{\infty} \ell(k) \kappa^{1 / r}\left(\delta^{-1} q_{2}^{-k} \varepsilon\right)^{-1 / r}
$$

of terms in the approximation 8.5 results with the same summation formula.

The operators $T_{k, \ell}$ map a Gauss function to a sum of $M / 2$ Gauss functions, where

$$
M=4\left(K N+\frac{(N-1) N}{2}\right)
$$

is the quadruple of the number of the interaction terms between the electrons and the nuclei and the electrons among each other and $K$ is the number of the nuclei. The estimates from Lemma 8.1 suggest therefore to choose

$$
\delta=\frac{1}{M^{r}}\left(\frac{1-q_{1}}{1+q_{1}}\right)^{2 r}, \quad \alpha \leq \frac{1}{2 M^{r}}\left(\frac{1-q_{1}}{1+q_{1}}\right)^{2 r+2} .
$$

The error estimate $(8.6)$ then reduces to

$$
\left\|\sum_{k=0}^{\infty} \sum_{\ell=1}^{\ell(k)} T_{k, \ell} u-\sum_{k=0}^{\infty} \sum_{\ell=1}^{\ell(k)} \sum_{j=1}^{n_{k}} T_{k, \ell} u_{j}\right\|_{1} \leq \frac{\varepsilon}{2},
$$


and the number of Gauss functions in the approximation $(8.5)$ is bounded by

$$
\frac{M}{2} \sum_{k=0}^{\infty} \sum_{\ell=1}^{\ell(k)} n_{k} \leq \frac{1}{2}\left(\frac{\kappa}{\varepsilon}\right)^{1 / r}
$$

Thus our goal is reached and we have shown that $\widetilde{T} u$ can be approximated by half number of Gauss functions with double accuracy, provided the width of the smoothing kernel (3.5) is sufficiently small, such that the condition on $\alpha$ from 8.9 holds. This condition limits the size of $\alpha$ the more the larger the approximation order $r$ becomes. In the limit $r=0$, it turns into

$$
\alpha \leq \frac{1}{2}\left(\frac{1-q}{1+q}\right)^{2} .
$$

It implies by (7.33) therefore the estimate $\|\widetilde{T}\|_{1} \leq 1 / 2$ for the operator $\widetilde{T}$ from the space $H^{1}$ into itself and thus ensures the convergence of the series 8.1 in $H^{1}$.

We have assumed that the number $n(\varepsilon)$ of terms in 8.2 needed to reach an error of norm $\leq \varepsilon$ in the approximation of the given function $u$ increases when $\varepsilon$ decreases. A decreasing $\varepsilon$ thus means that further terms are added to the sum 8.5 . Sorting and numbering the single terms correspondingly, one gets therefore a new sequence of Gauss functions $w_{j}$ such that, for all $\varepsilon>0$,

$$
\left\|\widetilde{T} u-\sum_{j=1}^{n_{1}(\varepsilon)} w_{j}\right\|_{1} \leq \varepsilon, \quad n_{1}(\varepsilon) \leq \frac{1}{2}\left(\frac{\kappa}{2 \varepsilon}\right)^{1 / r} .
$$

The old situation is thus restored, but with a new function $n_{1}(\varepsilon)$ counting the number of Gauss functions needed to obtain a given accuracy. As the constant $\kappa$ from 8.2 does not enter into the bound for $\alpha$, this process can be iterated.

Lemma 8.2 Starting from a function $u \in H^{1}$ as in 8.2 , for every $v=1,2, \ldots$ and every $\varepsilon>0$, the function $\widetilde{T}^{v} u$ can be approximated by a linear combination of

$$
n_{v}(\varepsilon) \leq \frac{1}{2^{v}}\left(\frac{\kappa}{2^{v} \varepsilon}\right)^{1 / r}
$$

Gauss functions up to an $H^{1}$-error $\varepsilon$, provided $\alpha$ is chosen as in (8.9).

Such a small norm of the operator $\widetilde{T}$ as enforced by the condition from 8.9 to the constant $\alpha$ means that only very few terms $-\widetilde{T} f, \widetilde{T}^{2} f, \ldots$ need to be added to the right hand side of the equation $u+\widetilde{T} u=f$ to approximate its solution

$$
u=f-\widetilde{T} f+\widetilde{T}^{2} f-\ldots
$$

with high accuracy. The first term $-\widetilde{T} f$ explicitly depends on the distances of the electrons and covers two-particle interactions, the second then also three-particle interactions, and so on. Lemma 8.2 means that less and less Gauss functions are necessary to approximate these terms sufficiently well and leads to our final and concluding 
Theorem 8.1 Let $f$ be a function in $H^{1}$ and assume that there exists an infinite sequence of Gauss functions $g_{1}, g_{2}, \ldots$ such that, for every $\varepsilon>0$,

$$
\left\|f-\sum_{j=1}^{n(\varepsilon)} g_{j}\right\|_{1} \leq \varepsilon, \quad n(\varepsilon) \leq\left(\frac{\kappa}{\varepsilon}\right)^{1 / r},
$$

where $r$ is a given approximation order and $\kappa$ a constant that depends on $f$ but is independent of $\varepsilon$. The solution $u \in H^{1}$ of the equation

$$
u+\widetilde{T} u=f
$$

can then, for every $\varepsilon>0$, be approximated by a linear combination of

$$
n \leq 2\left(\frac{2 \kappa}{\varepsilon}\right)^{1 / r}
$$

Gauss functions up to an $H^{1}$-error $\varepsilon$, provided the width of the smoothing kernel (3.5) is sufficiently small in dependence of the approximation order $r$, that is, the constant $\alpha$ from (7.24) satisfies the condition from 8.9 .

Proof The proof is based on the representation

$$
(I+\widetilde{T})^{-1} f=\sum_{v=0}^{\infty}(-1)^{v} \widetilde{T}^{v} f
$$

and the approximation of the parts $\widetilde{T}^{v} f$ up to an error $\varepsilon_{v}=2^{-(v+1)} \varepsilon$. By Lemma 8.2 , the needed number of Gauss functions sums then up to a value not larger than

$$
\sum_{v=0}^{\infty} \frac{1}{2^{v}}\left(\frac{\kappa}{2^{v} \varepsilon_{v}}\right)^{1 / r}=2\left(\frac{2 \kappa}{\varepsilon}\right)^{1 / r}
$$

and the errors $\varepsilon_{v}$ to the target accuracy $\varepsilon$, provided the width of the smoothing kernel (3.5) is so small that the constant $\alpha$ satisfies the condition from 8.9.

We remark that the theorem can be generalized from $H^{1}$ to every space $H^{s}$ of order $0 \leq s \leq 1+\vartheta$, under the same condition to the constant $\alpha$. The proof starts from a generalization of Lemma 7.7 and Lemma 7.8, considering the $V_{k}$ as operators from $H^{s}$ to $H^{t}, t=s-(1+\vartheta)$, and the $G_{k} P$ correspondingly as operators from $H^{t}$ back to the space $H^{s}$ of interest, and proceeds then as before.

The given approximations of the solution 8.1 can also be seen from a different perspective, decomposing them not into single Gauss functions but into a much smaller number of generic building blocks. Let us call these building blocks elementary functions and let us assume that the right hand side $f$ is given as a series of elementary functions of level zero, say as a series of Gauss functions as considered so far, or, for example, a series of Slater determinants of three-dimensional Gaussian orbitals. If $g$ is an elementary function of level $v$, the application of the operators $T_{k, \ell}$ to $g$ yields elementary functions of level $v+1$. That is, the $\widetilde{T}^{v} f$ are composed of elementary functions of level $v$, and their approximations of finite subsets of these functions. The symmetry properties of the elementary functions with respect to the 
exchange of the electron positions are inherited from one level to the next. Starting from corresponding approximation properties of the series representing the right hand side, the total number of elementary functions needed to approximate the solution up to a given accuracy can be estimated in exactly the same way as this has been done here for Gauss functions, where the number of elementary functions halves again from one level to the next. The condition from 8.9 needs only to be replaced by the weaker and less restrictive condition

$$
\alpha \leq \frac{1}{2^{r+1}}\left(\frac{1-q_{1}}{1+q_{1}}\right)^{2 r+2}
$$

into which the system parameters and the eigenvalue enter only indirectly via the definition (7.24) of the constant $\alpha$. With this new condition to $\alpha$, Theorem 8.1 literally transfers to the present situation. The price to be paid is the more complex, problemdependent structure of the single building blocks. The compact representation and compression of these building blocks needs further investigation.

In view of our application to the electronic wavefunction $u$ under consideration, the solution of the equation (4.7), the condition to the width of the smoothing kernel $K$ means that a sufficiently large part of $u$ needs to be shifted to the right hand side $f=Q u$ of the equation. The $H^{1}$-distance between the wavefunction $u$ and its smoothed variant $Q u=K * u$ is by (4.10) of order $\gamma^{1 / 2}$. The $H^{1}$-distance of the wavefunction $u$ and the solution $\widetilde{u}$ of the perturbed equation (4.8):

$$
\widetilde{u}+\widetilde{T} \widetilde{u}=f, \quad f=Q u,
$$

behaves by (4.9), by (6.6) and (6.11), and by (5.10) and (5.11) in comparison like

$$
\lesssim \gamma^{1 / 2} h^{-1 / 2} \mathrm{e}^{-\pi^{2} / h}
$$

For small $h$, the gap between the basic accuracy of order $\gamma^{1 / 2}$ and the attainable accuracy thus rapidly widens to many orders of magnitude. The conclusion from Theorem 8.1 is therefore that the approximation of the quasi-exact solution $\widetilde{u}$ of the Schrödinger equation does not require a substantially larger number of terms than that of the smoothed variant $Q u$ of the true wavefunction. The question remains when this rather astonishing effect actually sets in and how far the also with the best possible choice of the parameter $\vartheta<1 / 2$ still very stringent condition on the width of the smoothing kernel can be relaxed.

\section{Epilogue. First steps toward a numerical procedure}

Many difficulties still have to be overcome on the way to a numerical method that fully exploits the approximation properties of the given class of Gauss and GaussHermite functions and that enables to compute such approximations efficiently. This begins with the mentioned problem how to incorporate the symmetry properties enforced by the Pauli principle and how to store such antisymmetrized functions in compact form; there is no such thing as Slater determinants. However, there are some 
basic components that will presumably be part of such methods. One is approximate inverse iteration, a procedure that evolved in recent years into a very popular method for the solution of the large matrix eigenvalue problems that arise from the discretization of linear selfadjoint elliptic partial differential equations. The analysis of such methods essentially started with the work of D'yakonov and Orekhov [9]. In a series of groundbreaking papers, Knyazev and Neymeyr analyzed these methods in great detail; we refer to [12] and the literature cited therein.

Approximate inverse iteration can be directly applied to operator equations in infinite dimensional spaces [16] and can best be understood in terms of the weak formulation of the eigenvalue problems. Let $\mathscr{H}$ be a Hilbert space that is equipped with the inner product $a(u, v)$ inducing the energy norm $\|u\|$, under which it is complete, and a further inner product $(u, v)$. Let the infimum of the Rayleigh quotient

$$
\lambda(u)=\frac{a(u, u)}{(u, u)}, \quad u \neq 0 \text { in } \mathscr{H},
$$

be an isolated eigenvalue $\lambda_{1}>0$ of finite multiplicity and let $\mathscr{E}_{1}$ be the assigned eigenspace, the finite dimensional space of all $u \in \mathscr{H}$ for which

$$
a(u, v)=\lambda_{1}(u, v), \quad v \in \mathscr{H},
$$

or equivalently $\lambda(u)=\lambda_{1}$ holds. The aim is the calculation of this eigenvalue, that is, the ground state energy of the system under consideration.

Let $\lambda_{2}>\lambda_{1}$ be the infimum of the Rayleigh quotient on the with respect to both inner products orthogonal complement of the eigenspace $\mathscr{E}_{1}$ for the eigenvalue $\lambda_{1}$. In cases like ours, $\lambda_{2}$ is also an isolated eigenvalue, but this is not truly needed. Given an element $u \in \mathscr{H}$ with norm $\|u\|_{0}=1$ and Rayleigh quotient $\lambda(u)<\lambda_{2}$, in inverse iteration in its original, exact version at first the solution $w \in \mathscr{H}$ of the equation

$$
a(w, v)=a(u, v)-\lambda(u)(u, v), \quad v \in \mathscr{H},
$$

is determined, which exists by the Riesz representation or the Lax-Milgram theorem and is unique. The current $u$ is then replaced by $u-w$. Since $a(u, w)=0$, the new element $u-w$ is different from zero so that $\lambda(u-w)$ is well defined and the process can be repeated with the normed version of $u-w$. The so iteratively generated sequence of Rayleigh quotients decreases then monotonously to the eigenvalue $\lambda_{1}$ and the iterates $u$ converge to an eigenvector or eigenfunction for this eigenvalue.

In the approximate version of the method, the solution $w$ of equation (9.3) is replaced by an approximation $\widetilde{w} \in \mathscr{H}$ for which an error estimate

$$
\|\widetilde{w}-w\| \leq \delta\|w\|
$$

holds, where $\delta<1$ is a fixed constant that controls the accuracy. Then $u-\widetilde{w} \neq 0$, so that the process can proceed with the normed version $u^{\prime}$ of $u-\widetilde{w}$ as new iterate. No assumption on the origin of $\widetilde{w}$ is needed. It can, for example, be the element of best approximation of $w$ in a finite dimensional subspace of $\mathscr{H}$, an iteratively calculated approximation of this element, or anything else wherever it comes from. 
A simple, albeit surely not optimal analysis of this variant along the lines given in the original paper of D'yakonov and Orekhov can be found in [22]. Main result is that under the given assumptions, and if in particular already $\lambda(u)<\lambda_{2}$, the estimate

$$
\lambda\left(u^{\prime}\right)-\lambda_{1} \leq q(\lambda(u))\left(\lambda(u)-\lambda_{1}\right)
$$

holds, where $q(\lambda)$ is the on the interval $\lambda_{1} \leq \lambda \leq \lambda_{2}$ strictly increasing function

$$
q(\lambda)=1-\frac{\left(1-\delta^{2}\right) \lambda\left(\lambda_{2}-\lambda\right)^{2}}{\lambda_{2}^{2} \lambda+\left(1-\delta^{2}\right)\left(\lambda_{2}-\lambda\right)^{2}\left(\lambda-\lambda_{1}\right)} .
$$

If one starts therefore with a normed $u=u_{0}$ in $\mathscr{H}$ with Rayleigh quotient $\lambda\left(u_{0}\right)<\lambda_{2}$ and generates as described a sequence of normed $u_{k}$, the Rayleigh quotients $\lambda\left(u_{k}\right)$ decrease strictly to the minimum eigenvalue $\lambda_{1}$ or become stationary there. Moreover, one can show that the iterates $u_{k}$ converge to an eigenvector for the eigenvalue $\lambda_{1}$.

To apply this form of inverse iteration to our eigenvalue problem (2.6), we have first to shift the Hamiltonian and to replace the bilinear form (2.5) by a bilinear form

$$
a(u, v)=\int\{\nabla u \cdot \nabla v+V u v+\mu u v\} \mathrm{d} x
$$

on $\mathscr{H}=H^{1}$, with $\mu>0$ a still to be determined constant value. The original eigenvalues $\lambda$ turn then into $\lambda+\mu$; the eigenfunctions themselves remain untouched. The term $a(u, u)$ can, according to Lemma 2.1 be estimated from above and below by

$$
\|\nabla u\|_{0}^{2} \pm \theta\|\nabla u\|_{0}\|u\|_{0}+\mu\|u\|_{0}^{2}
$$

where $\|u\|_{0}$ is the $L_{2}$-norm and $\theta$ the norm (3.11) of the potential $V$, understood as operator from $H^{1}$ to $L_{2}$. Making the expressions (9.8) extremal under the constraint $\|\nabla u\|_{0}^{2}+\mu\|u\|_{0}^{2}=1$, one can therefore estimate $a(u, u)$ from below and above by

$$
\left(1-\frac{\theta}{2 \sqrt{\mu}}\right) b(u, u) \leq a(u, u) \leq\left(1+\frac{\theta}{2 \sqrt{\mu}}\right) b(u, u)
$$

in terms of the inner product

$$
b(u, v)=\int\{\nabla u \cdot \nabla v+\mu u v\} \mathrm{d} x
$$

on $H^{1}$, now without the potential part. The shifted bilinear form 9.7 fits therefore for values $\mu>\theta^{2} / 4$ into the described framework of approximate inverse iteration.

An obvious choice for the approximation of the solution $w \in H^{1}$ of equation (9.3) is in the given context the solution $\widetilde{w} \in H^{1}$ of the equation

$$
b(\widetilde{w}, v)=a(u, v)-\lambda(u)(u, v), \quad v \in H^{1} .
$$

By the definition of $w$ and $\widetilde{w}$ and again by Lemma 2.1 then the estimate

$$
b(\widetilde{w}-w, \widetilde{w}-w)=(V w, \widetilde{w}-w) \leq \theta\|\nabla w\|_{0}\|\widetilde{w}-w\|_{0}
$$


holds. With the help of (9.9), finally the energy norm estimate

$$
\|\widetilde{w}-w\| \leq \sqrt{c(\eta)} \eta\|w\|
$$

in terms of $\eta=\theta / \sqrt{\mu}$ and the function $c(\eta)=(2+\eta) /(2-\eta)$ follows. That is, for sufficiently large constants $\mu$ the basic condition (9.4) is fulfilled.

Now we have reached the point at which the Gauss functions come into play. With $u$ given, the new approximation reads, before normalization, in operator form

$$
u-\widetilde{w}=u-(-\Delta+\mu)^{-1}(-\Delta u+\mu u+V u-\lambda(u) u) .
$$

The idea is to approximate the operators $(-\Delta+\mu)^{-1}$ and $V$ in the discussed manner or similarly by means of Gauss functions. Gauss functions are then again mapped to series of Gauss functions, which have to be truncated appropriately. The residual

$$
-\Delta u+\mu u+V u-\lambda(u) u
$$

actually does not depend on the choice of the constant $\mu$, which enters into the process only via the inverse of the shifted Laplace operator. It needs to be approximated with high accuracy to fulfill the condition (9.4) on the accuracy of the approximation $\widetilde{w}$. The requirements for the approximation of the inverse of the shifted Laplace operator are in comparison modest. The price to be paid is that the degree of the polynomial part of the Gaussians increases due the Laplace part in the residual from one step to the next. Starting from the representation

$$
u-\widetilde{w}=(-\Delta+\mu)^{-1}(\lambda(u) u-V u)
$$

of the new iterate before normalization this can be avoided, but conversely then also the inverse of the shifted Laplacian has to be approximated with high accuracy. The big unsolved question with both variants is how to truncate the intermediate series of Gauss functions to keep the number of terms on a computationally still feasible level, without sacrificing the accuracy or lowering it too much.

\section{Appendix. Remarks on interpolation}

In Sect.7 more precisely in the proof of Lemma 7.7 and 7.8, we have used some results from interpolation theory. The interpolation between Banach and Hilbert spaces is a large and well established field of functional analysis and the theory of function spaces. A standard reference is [3]. A more condensed presentation coming closer to our needs can be found in [15]. We are in the lucky situation that the proof of the mentioned two lemmata requires only the interpolation within the space of rapidly decreasing functions, a fact that simplifies the argumentation a lot and enables us to derive the necessary results in a few lines.

The key is the representation of the norms on the space $\mathscr{S}$ of the real-valued, rapidly decreasing functions in terms of the $K$-functionals

$$
K\left(t, u, \vartheta_{1}, \vartheta_{2}\right)=\inf _{v \in \mathscr{S}}\left\{\|u-v\|_{\vartheta_{1}}^{2}+t^{2}\|v\|_{\vartheta_{2}}^{2}\right\}^{1 / 2}, \quad \vartheta_{1}<\vartheta_{2}
$$

which can be considered as more refined smoothness measures. 
Lemma Let $\vartheta_{1}<\vartheta_{2}, 0<s<1$, and $\vartheta=\vartheta_{1}+s\left(\vartheta_{2}-\vartheta_{1}\right)$. For all $u \in \mathscr{S}$ then

$$
\int_{0}^{\infty}\left[t^{-s} K\left(t, u, \vartheta_{1}, \vartheta_{2}\right)\right]^{2} \frac{\mathrm{d} t}{t}=\int_{0}^{\infty} \frac{t^{1-2 s}}{1+t^{2}} \mathrm{~d} t\|u\|_{\vartheta}^{2}
$$

Proof The expression whose infimum is sought reads in Fourier representation

$$
\int\left\{\left(1+|\omega|^{2}\right)^{\vartheta_{1}}|\widehat{u}(\omega)-\widehat{v}(\omega)|^{2}+t^{2}\left(1+|\omega|^{2}\right)^{\vartheta_{2}}|\widehat{v}(\omega)|^{2}\right\} \mathrm{d} \omega
$$

The integrand is, with given $\widehat{u}(\omega)$, pointwise minimized by the value

$$
\widehat{v}(\omega)=\frac{\widehat{u}(\omega)}{1+t^{2}\left(1+|\omega|^{2}\right)^{\vartheta_{2}-\vartheta_{1}}} .
$$

This expression defines another real-valued, rapidly decreasing function $v$ at which the infimum is attained. Inserting this function above, we get a closed representation of the $K$-functional $K\left(t, u, \vartheta_{1}, \vartheta_{2}\right)$ of $u$ in terms of the Fourier transform of $u$ :

$$
K\left(t, u, \vartheta_{1}, \vartheta_{2}\right)^{2}=\int \frac{t^{2}\left(1+|\omega|^{2}\right)^{\vartheta_{2}-\vartheta_{1}}}{1+t^{2}\left(1+|\omega|^{2}\right)^{\vartheta_{2}-\vartheta_{1}}}\left(1+|\omega|^{2}\right)^{\vartheta_{1}}|\widehat{u}(\omega)|^{2} \mathrm{~d} \omega
$$

The proposition follows from this representation with Fubini's theorem.

Let us assume now that we have a linear operator $T: \mathscr{S} \rightarrow \mathscr{S}$ and that for all $u \in \mathscr{S}$

$$
\|T u\|_{\vartheta_{1}^{\prime}} \leq c\|u\|_{\vartheta_{1}}, \quad\|T u\|_{\vartheta_{2}^{\prime}} \leq c\|u\|_{\vartheta_{2}}
$$

where $\vartheta_{1}<\vartheta_{2}$ and $\vartheta_{1}^{\prime}<\vartheta_{2}^{\prime}$ are two arbitrarily given pairs of real numbers.

Lemma Let $0 \leq s \leq 1, \vartheta=\vartheta_{1}+s\left(\vartheta_{2}-\vartheta_{1}\right)$, and $\vartheta^{\prime}=\vartheta_{1}^{\prime}+s\left(\vartheta_{2}^{\prime}-\vartheta_{1}^{\prime}\right)$. For all real-valued, rapidly decreasing functions $u$ then, with the same constant,

$$
\|T u\|_{\vartheta^{\prime}} \leq c\|u\|_{\vartheta}
$$

Proof For $s=0$ and $s=1$, the proposition already holds by assumption. For the remaining values in between it follows immediately from the estimate

$$
K\left(t, T u, \vartheta_{1}^{\prime}, \vartheta_{2}^{\prime}\right) \leq c K\left(t, u, \vartheta_{1}, \vartheta_{2}\right)
$$

for the $K$-functionals of $u$ and $T u$ assigned to the given norms and the representation above of the two intermediate norms in terms of these $K$-functionals. 


\section{References}

1. Bachmayr, M., Chen, H., Schneider, R.: Error estimates for Hermite and even-tempered Gaussian approximations in quantum chemistry. Numer. Math. 128, 137-165 (2014)

2. Bachmayr, M., Dahmen, W.: Adaptive near-optimal rank tensor approximation for high-dimensional operator equations. Found. Comp. Math. 15, 839-898 (2015)

3. Bergh, J., Löfström, J.: Interpolation Spaces, Die Grundlehren der mathematischen Wissenschaften in Einzeldarstellungen, vol. 223. Springer, Berlin Heidelberg New York (1976)

4. Beylkin, G., Monzón, L.: Approximation by exponential sums revisited. Appl. Comput. Harmon. Anal. 28, 131-149 (2010)

5. Braess, D.: Asymptotics for the approximation of wave functions by exponential sums. J. Approx. Theor. 83, 93-103 (1995)

6. Braess, D., Hackbusch, W.: Approximation of $1 / x$ by exponential sums in $[1, \infty)$. IMA J. Numer. Anal. 25, 685-697 (2005)

7. Braess, D., Hackbusch, W.: On the efficient computation of high-dimensional integrals and the approximation by exponential sums. In: R. DeVore, A. Kunoth (eds.) Multiscale, Nonlinear and Adaptive Approximation. Springer, Berlin Heidelberg (2009)

8. Dahmen, W., DeVore, R., Grasedyck, L., Süli, E.: Tensor-sparsity of solutions to high-dimensional elliptic partial differential equations. Found. Comp. Math. 16, 813-874 (2016)

9. D’yakonov, E., Orekhov, M.: Minimization of the computational labor in determining the first eigenvalues of differential operators. Mat. Zametki 27, 795-812 (1980). In Russian, English translation: Math. Notes 27 (1980), pp. 382-391

10. Hackbusch, W.: Tensor Spaces and Numerical Tensor Calculus, Springer Series in Computational Mathematics, vol. 42. Springer, Heidelberg Dordrecht London New York (2012)

11. Helgaker, T., Jørgensen, P., Olsen, J.: Molecular Electronic Structure Theory. John Wiley \& Sons, Chichester (2000)

12. Knyazev, A.V., Neymeyr, K.: Gradient flow approach to geometric convergence analysis of preconditioned eigensolvers. SIAM J. Matrix Anal. Appl. 31, 621-628 (2009)

13. Kreusler, H.C., Yserentant, H.: The mixed regularity of electronic wave functions in fractional order and weighted Sobolev spaces. Numer. Math. 121, 781-802 (2012)

14. Kutzelnigg, W.: Theory of the expansion of wave functions in a Gaussian basis. Int. J. Quant. Chem. 51, 447-463 (1994)

15. McLean, W.: Strongly Elliptic Systems and Boundary Integral Equations. Cambridge University Press, Cambridge (2000)

16. Rohwedder, T., Schneider, R., Zeiser, A.: Perturbed preconditioned inverse iteration for operator eigenvalue problems with applications to adaptive wavelet discretization. Adv. Comput. Math. 34, 43-66 (2011)

17. Scholz, S.: Zur Approximation elektronischer Wellenfunktionen durch anisotrope Gauß-Funktionen. Doctoral thesis, Technische Universität Berlin (2016)

18. Yafaev, D.: Sharp constants in the Hardy-Rellich inequalities. J. Funct. Anal. 168, 121-144 (1999)

19. Yserentant, H.: On the regularity of the electronic Schrödinger equation in Hilbert spaces of mixed derivatives. Numer. Math. 98, 731-759 (2004)

20. Yserentant, H.: Regularity and Approximability of Electronic Wave Functions, Lecture Notes in Mathematics, vol. 2000. Springer, Heidelberg Dordrecht London New York (2010)

21. Yserentant, H.: The mixed regularity of electronic wave functions multiplied by explicit correlation factors. ESAIM: M2AN 45, 803-824 (2011)

22. Yserentant, H.: A note on approximate inverse iteration. arXiv:1611.04141 [math.NA] (2016) 